\title{
Outcomes of Glaucoma Drainage Device Surgery in Eyes with Treated Uveal Melanoma
}

\author{
Nima Fatehi Tara A. McCannel JoAnn Giaconi Joseph Caprioli Simon K. Law
}

Kouros Nouri-Mahdavi

Stein Eye and Doheny Eye Institutes, David Geffen School of Medicine, University of California Los Angeles, Los Angeles, CA, USA

\section{Keywords}

Uveal melanoma - Glaucoma - Intraocular pressure ·

Metastasis · Glaucoma drainage device

\section{Abstract}

Aim: We report patient safety and intraocular pressure (IOP) control after placement of a glaucoma drainage device (GDD) in eyes with a history of treated malignant uveal melanoma. Methods: A retrospective review of the records of patients with uveal melanoma was performed. Outcomes were local tumor recurrence, rate of metastases, and tonometric success, based on survival curves, defined as IOP $<21 \mathrm{~mm} \mathrm{Hg}$. Results: Eleven eyes with choroidal melanoma, 4 with iris melanoma, and 1 with ciliary body melanoma were followed for a median (interquartile range) of 2.1 (1.13.2) years. Two subjects developed liver metastases; one had monosomy 3 and tumor gene expression profile class 2 . The other case with ciliary body melanoma was negative for monosomy 3. There were no cases of local treatment failure. Mean preoperative IOP decreased from $30.5 \pm 7.7$ to $15.9 \pm$ $8.1 \mathrm{~mm} \mathrm{Hg}$ at 1 year after surgery (1-year success rate $80 \%$ ). Conclusions: Our case series with a median follow-up of

\section{KARGER}

(c) 2018 S. Karger AG, Basel

E-Mail karger@karger.com

www.karger.com/oop
2 years shows that placing a GDD in patients with treated uveal melanoma does not expose patients to greater risk of local or extraocular recurrence. A larger series and longer follow-up time are required to fully evaluate the safety of GDDs in this clinical scenario.

(c) 2018 S. Karger AG, Basel

\section{Introduction}

Melanoma is a malignant tumor that may develop in organs and tissues harboring melanocytes, such as skin, eye, and mucosa. The uveal tissue of the eye is the second most common site for primary melanoma. Among uveal melanomas, melanoma of the choroid (69-90\%) is the most common site followed by the iris $(5-8 \%)$ and ciliary body $(4-7 \%)[1-4]$. We have recently reported the incidence of secondary open-angle glaucoma after brachytherapy to be $8.6 \%$ in a series of 374 uveal melanoma patients [Kim et al., manuscript under revision]; the incidence of neovascular glaucoma in the same cohort was $6.7 \%$. The risk factors for secondary open-angle glaucoma included older age, larger tumor size, iris location, and 
higher baseline intraocular pressure (IOP). The risk factors for neovascular glaucoma were larger tumor size, pseudophakia, and greater extent of retinal ischemia on wide-field fluorescein angiography. Other studies have reported the incidence of glaucoma in eyes with treated uveal melanoma to vary from 3 to $59 \%$ [1-3, 5-7].

Management of glaucoma in eyes with uveal melanoma, however, remains controversial. Uncontrolled glaucoma from neovascularization is the main reason for enucleation of the eye in patients with anterior uveal melanoma [8-10]. Conventional modalities to control IOP include medical treatment, trabeculectomy, or placement of a glaucoma drainage device (GDD) and ciliary body ablation [1]. Medical therapy alone is frequently inadequate for controlling IOP in eyes with treated uveal melanoma $[11,12]$. In contrast to eyes with other forms of secondary glaucoma, glaucoma filtration surgery and placement of a GDD are not considered first-line approaches in eyes harboring a melanoma, despite tumor control with radiotherapy. Fear of both local and distant extraocular seeding of the tumor may prevent melanoma eyes from receiving conventional glaucoma filtration surgery for optimal IOP control $[10,13-16]$. There is scant information on the outcomes and potential risks of filtering surgery in eyes with melanoma. Sharkawi et al. [17] reported that placement of a Baerveldt glaucoma implant in eyes with anterior uveal melanoma was associated with minimal complications up to 2 years after surgery.

The goal of the current study is to report our center's outcomes and ocular and systemic complications after placement of a GDD in patients with a history of treated uveal melanoma.

\section{Methods}

This study is a retrospective cohort study describing the outcomes and complications of placement of a GDD in consecutive patients who were previously treated for uveal melanoma and in whom local tumor control was established. The subjects were identified through a database search at the Stein Eye institute, University of California Los Angeles.

Inclusion criteria included a history of GDD placement in an eye with treated uveal melanoma and a minimum follow-up of 3 months after GDD placement. A total of 670 patients with a history of treated uveal melanoma were identified, among whom 16 met the above inclusion criteria. The collected data from the medical record were age, sex, race, visual acuity, location and size of the tumor at baseline, IOP at baseline, glaucoma mechanism, IOP before GDD placement, IOP at 3, 6, and 12 months after placement of the glaucoma drainage device and 6-monthly intervals, cytopathology and molecular prognostic testing result from fine needle aspiration biopsy, and metastatic outcomes.

Outcomes of GDD Surgery in Eyes with

Treated Uveal Melanoma

\section{Placement of Plaque}

Iodine-125 plaque brachytherapy was used for treatment of uveal melanoma. The dosage of the radiation was determined based on the American Association of Physicists in Medicine guidelines [18]. The technique for plaque placement has been previously described and involved $360^{\circ}$ peritomy followed by identification and isolation of the four rectus muscles with 2-0 silk ties. The anterior margin of the tumor was then identified by transpupillary transillumination. If the rectus muscles or their insertions prevented implantation of the plaque, they were disinserted and reattached at the time of plaque removal. The location of the plaque with respect to the tumor was confirmed and optimized with intraoperative ultrasonography [19]. Fine needle aspiration biopsy of the tumor was performed for cytology and molecular prognostic studies.

\section{Vitrectomy with Silicone Oil Placement for Radiation}

\section{Attenuation}

In cases where the vitreous was substituted with silicone oil as a radiation-attenuating agent, a standard three-port 23-gauge complete pars plana vitrectomy was performed after ultrasoundguided plaque placement. The posterior hyaloid was elevated and the vitreous removed to the level of the retinal periphery with $360^{\circ}$ scleral indentation. A fluid-air exchange was performed followed by an air-silicone oil exchange (1,000 centistokes). The sclerotomy sites were sutured $[20,21]$.

\section{Surgical Technique for GDD}

A $90^{\circ}$ peritomy was created in the quadrant of the eye with no melanoma involvement and two 4-mm radial incisions were made extending posteriorly at both ends of the limbal incision. The rectus muscles were identified and Tenon's capsule and conjunctiva were dissected off the sclera in the quadrant of interest extending posteriorly behind the equator. An Ahmed glaucoma valve (FP7) was primed with balanced salt solution and placed in the superotemporal quadrant underneath Tenon's capsule. In cases where a Baerveldt glaucoma implant $\left(250 \mathrm{~mm}^{2}\right)$ was used, tube patency was checked with a 25-gauge cannula connected to a balanced salt solution syringe, and the plate was then placed under the recti muscles. Two 8-0 nylon or 7-0 polypropylene sutures were used to fix the GDD plate onto the sclera. A 22-gauge needle was used to create a needle track for inserting the tube. For Ahmed glaucoma valve cases, the anterior chamber was filled with cohesive viscoelastic, and the tube was then inserted into the anterior chamber and its position was deemed appropriate. The Baerveldt glaucoma implant tube was ligated with a 7-0 polyglactin suture about 6-8 $\mathrm{mm}$ from the limbus, and two venting slits were created anterior to the ligating suture. The tube was covered with a pericardial graft (Tutoplast; IOP Ophthalmics, Costa Mesa, CA, USA) or split thickness corneal half-moon (VisionGraft; Tissue Banks International, Baltimore, MD, USA). The conjunctiva was closed with 9-0 polyglactin sutures.

\section{Molecular Prognostic Test Results}

The cytogenetic characteristics of tumors cells were assessed by three methods. Cytogenetic analyses were performed by fluorescence in situ hybridization (FISH) for the centromere of chromosome 3; the details have been described in a previous report [22]. The biopsies were also assessed for prognosis by multiplex ligation-dependent probe amplification (MLPA) and gene ex- 


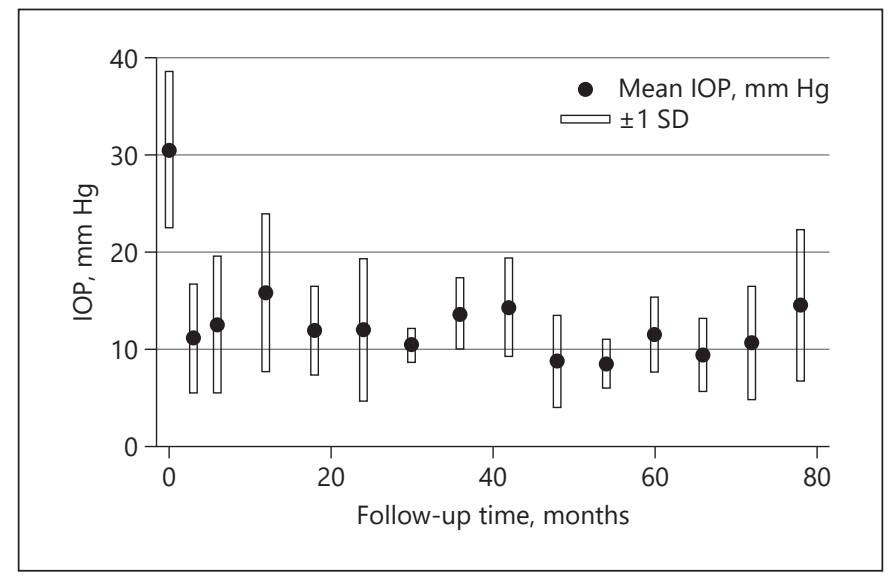

Fig. 1. The course of IOP after placement of a glaucoma drainage device in eyes with ocular melanoma. The bars represent \pm 1 SD. $\mathrm{IOP}$, intraocular pressure; SD, standard deviation.

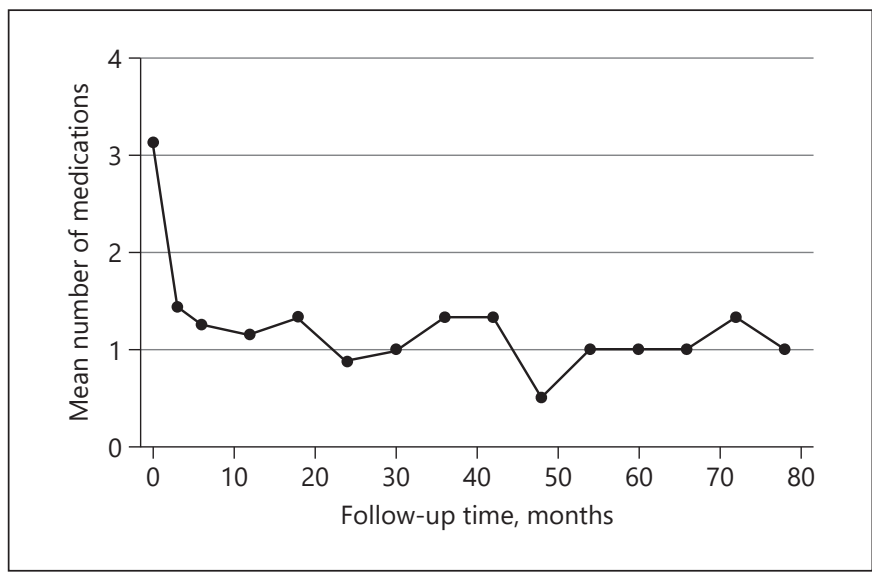

Fig. 2. The course of the number of medications after placement of a glaucoma drainage device in eyes with ocular melanoma.

Table 1. Demographic and clinical characteristics of the study patients

\begin{tabular}{lc}
\hline Age at the time of melanoma treatment, years (mean \pm SD) & $65.1 \pm 10.1$ \\
Sex (male/female) & $6 / 10$ \\
Ethnicity (Caucasian/African American) & $15 / 1$ \\
Location of uveal melanoma (choroid/iris/ciliary body) & $10 / 5 / 1$ \\
Media time between melanoma treatment and GDD surgery, years (range) & $1.0(0.1-12)$ \\
Median follow-up after treatment of melanoma, years (IQR) & $3.5(2.3-5.3)$ \\
Median follow-up after GDD surgery, years (IQR) & $2.1(1.1-3.2)$ \\
Number of patient with metastasis & 2 \\
Number of patients deceased from metastatic disease & 1
\end{tabular}

GDD, glaucoma drainage device; IQR, interquartile range; SD, standard deviation.

pression profile (GEP) assay for uveal melanoma. These tests were performed depending on their availability at the time of biopsy.

\section{Statistical Analyses}

Measures of distribution were used to describe numerical variables such as age, IOP, follow-up time after melanoma treatment or after GDD insertion, and number of glaucoma medications before and after surgery. We considered glaucoma surgery to have failed when IOP was $\geq 21 \mathrm{~mm} \mathrm{Hg}$ or $<6 \mathrm{~mm} \mathrm{Hg}$ on two consecutive visits, if the patient needed additional glaucoma surgery, or if the eye lost light perception vision. We used Kaplan-Meier survival curves to estimate the tonometric success rate of GDD surgery.

\section{Results}

Sixteen patients met the inclusion criteria for the study and were included in the final analyses; 15 subjects had 16 Ahmed glaucoma valves placed and 1 subject underwent
Baerveldt glaucoma implant placement. Table 1 shows the demographic and clinical characteristics of the study patients. The mean age $( \pm \mathrm{SD})$ of study patients was $65.1 \pm 10.1$ years at the time of melanoma treatment. There were 11 cases of choroidal melanoma, 4 eyes with iris melanoma, and 1 case of ciliary body melanoma involving the iris. The median (interquartile range) followup times after melanoma treatment and after GDD placement were $3.5(2.3-5.3)$ and $2.1(1.1-3.2)$ years, respectively. Mean $( \pm$ SD) IOP before GDD placement was 30.5 $\pm 8.0 \mathrm{~mm} \mathrm{Hg}$ and decreased to $15.9 \pm 8.1 \mathrm{~mm} \mathrm{Hg}$ at 1 year and to $14.0 \pm 6.0 \mathrm{~mm} \mathrm{Hg}$ at last follow-up. The baseline average number of medications before GDD placement was 3.2 (range $0-4$ ). The courses of IOP and number of medications after placing the GDD are shown in Figures 1 and 2, respectively.

Table 2 shows the main characteristics and the course of glaucoma and melanoma in individual patients in our 
study. Of the 16 patients, 12 had secondary angle-closure glaucoma, 2 had primary open-angle glaucoma, and 2 had been diagnosed with neovascular glaucoma. Fifteen subjects received treatment with iodine- 125 plaque for treatment of melanoma, and 1 patient had partial iridectomy for iris melanoma. Table 3 shows the history of intraocular surgeries of individual patients before, concomitant with, or after melanoma treatment. Six patients had pars plana vitrectomy with 1,000 centistokes silicone oil placement for radiation attenuation; 5 of them also underwent cataract extraction with intraocular lens placement, and 1 patient had cataract surgery alone with plaque insertion.

\section{Genetic Characteristics}

Fine needle aspiration was performed in all patients except for patient 7, who had undergone partial iridectomy. Six samples were not adequate for evaluation. FISH cytogenetic analyses for 9 out of 15 cases were available; 7 patients had negative result for monosomy 3 and 2 patients had positive results. MLPA was available for 4 patients; 2 patients had monosomy 3 , and 2 patients had normal results. The GEP assay for uveal melanoma was available for 7 patients, of whom 2 had class 2 GEP, 1 showed class 1B GEP, and 4 demonstrated class 1A GEP (Table 2).

\section{Metastatic Outcomes}

Two cases developed uveal melanoma metastases to the liver. The first patient (case 3 ) had a primary ciliary body melanoma. Metastases were detected 39 months after brachytherapy and 21 months after insertion of the drainage device. The patient expired 1 month after detection of metastases due to widespread metastatic disease. Tumor cytogenetic data obtained from fine needle aspiration biopsy revealed disomy 3 with FISH testing; data for the gene expression assay for uveal melanoma and MLPA were not obtained. The second patient with metastatic disease (case 12) had choroidal melanoma treated with brachytherapy plaque and concomitant phacoemulsification, intraocular lens placement, vitrectomy, and placement of silicone oil for radiation attenuation. He was diagnosed with metastatic liver lesions 14 months after melanoma treatment and 10 months after Ahmed glaucoma valve placement. The patient was treated with chemotherapy and radiation for metastatic disease. Tumor cytogenetic information obtained by fine needle aspiration was positive for chromosome 3 monosomy with both FISH and MLPA testing. GEP testing revealed a class 2 test result. One patient (case 6) had a history of trabecu-

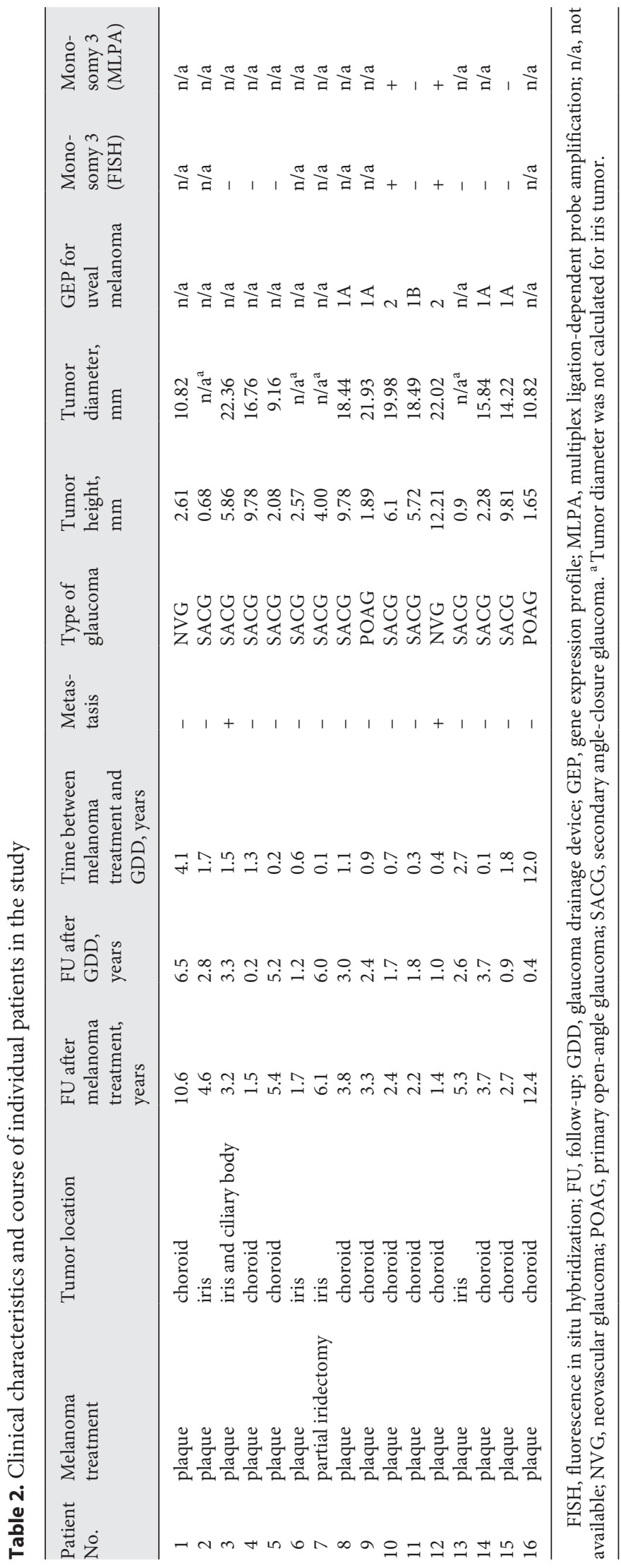

Ocul Oncol Pathol 2019;5:20-27 DOI: $10.1159 / 000488056$
Outcomes of GDD Surgery in Eyes with

Treated Uveal Melanoma 
Table 3. Intraocular surgeries before, concomitant with, or after melanoma treatment

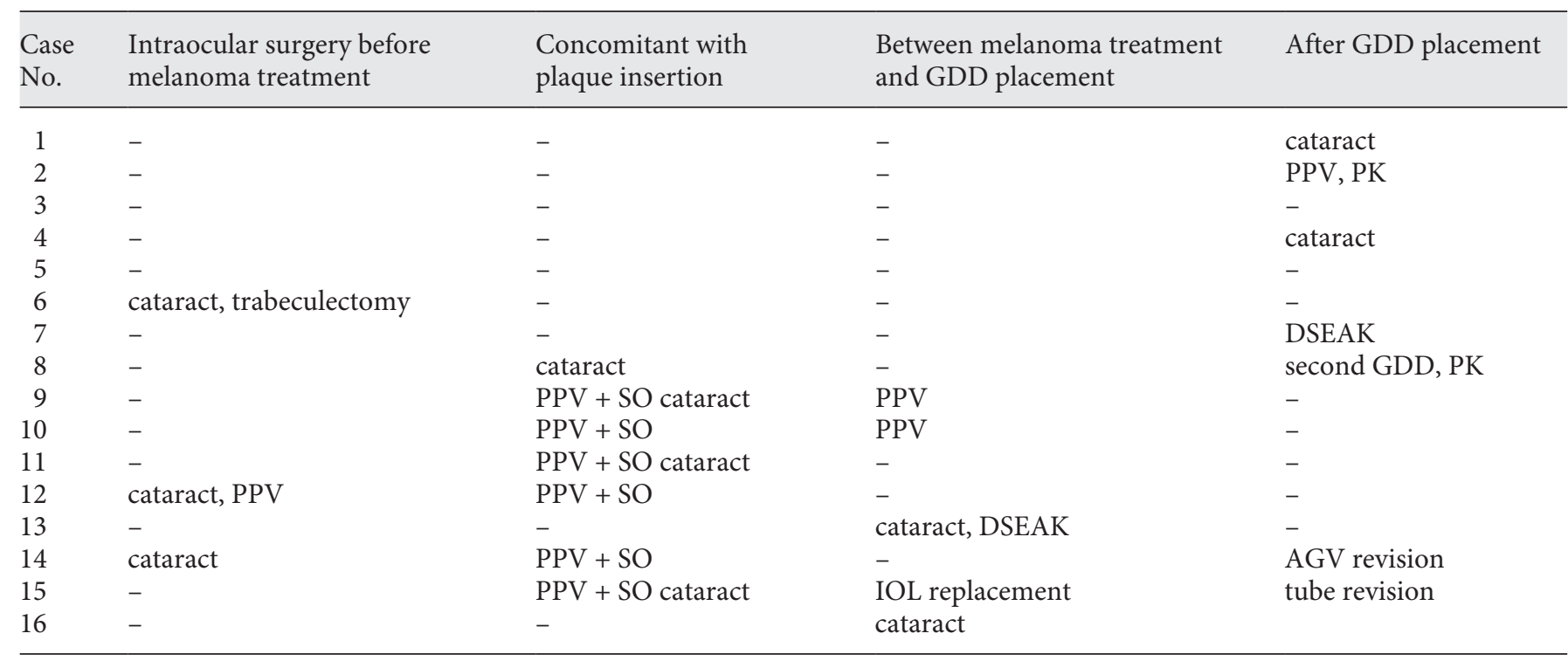

AGV, Ahmed glaucoma valve; DSEAK, Descemet stripping automated keratoplasty; GDD, glaucoma drainage device; IOL, intraocular lens; PK, penetrating keratoplasty; PPV, pars plana vitrectomy; SO, silicone oil.

lectomy before treatment of the iris melanoma. At the time of plaque insertion, evidence of tumor was observed at the site of previous trabeculectomy. For this patient, the plaque was placed over the whole ciliary body plane including the trabeculectomy site.

\section{IOP Control and Success Rate of GDD Surgery}

We used Kaplan-Meier survival curves to estimate the success rate of GDD surgery in our patient sample. The success rate was $80 \%$ (95\% confidence interval [CI] 53$94 \%)$ at 1 year, $75 \%$ (95\% CI $45-90 \%)$ at 2 years, and $75 \%$ (95\% CI $45-90 \%)$ at 5 years of follow-up, based on our success criterion of IOP $<21 \mathrm{~mm} \mathrm{Hg}$ with or without medications (Fig. 3). One patient (case 8 ) had a second Ahmed glaucoma valve 24 months after the first one because of uncontrolled IOP on three antihypertensive medications. One patient required tube revision due to tube exposure.

\section{Discussion}

We report on 16 patients who underwent placement of a GDD for inadequately controlled glaucoma following plaque brachytherapy for uveal melanoma. There were no cases of local tumor dissemination to the orbit. Two patients eventually developed metastases of the uveal

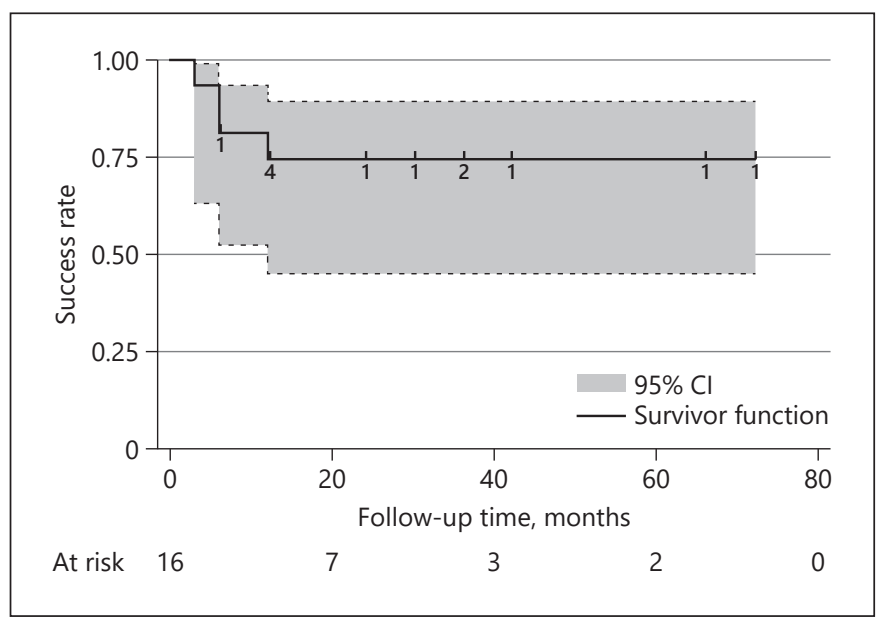

Fig. 3. Kaplan-Meier curve demonstrating the tonometric success rate (with 95\% CIs) after placement of a glaucoma drainage device in eyes with ocular melanoma. CI, confidence interval. At risk, number of patients at risk.

melanoma. These patients had large tumors and poor prognostic molecular genetic test results of the primary uveal melanoma.

During the past decade, the use of GDDs for controlling IOP in various forms of glaucoma has increased [2325]. However, there remains concern and significant con- 
troversy regarding glaucoma surgery in eyes with an intraocular tumor due to fear of both orbital and systemic tumor dissemination, increasing metastatic risk $[1,9,10$, $13,15,26]$. Although there are published cases of tumor dissemination following glaucoma surgery, in almost all cases local ocular tumor control had not been achieved $[13-16,27]$. Therefore, there are possibly risks in cases where the primary uveal melanoma has not been adequately treated. However, our data support the assumption that in cases in whom the primary tumor has been definitively treated (i.e., eyes with no evidence of tumor growth at the time of GDD placement), the risk to the patient following invasive glaucoma surgery is not greater than clinical risk factors, namely tumor size and molecular prognostic profile, would suggest. Treatment of glaucoma in eyes with intraocular tumors, which is often caused by multiple mechanisms (incidence rate of $15.3 \%$ [Kim et al., manuscript under revision]) [1,3,28], may be challenging, and medical therapy, laser trabeculoplasty, and cyclophotocoagulation may not lead to adequate IOP control $[11,29,30]$. Cyclophotocoagulation is a treatment that may result in eventual phthisis if repeated more than once; therefore, offering cyclophotocoagulation may be minimally better than enucleation for ocular preservation [31]. Previous studies found that the most common cause of enucleation in eyes treated for ocular melanoma was refractory glaucoma $[9,10,32,33]$.

With regard to IOP control, our patient cohort had success rates of approximately $80 \%$ at 1 year and $75 \%$ at 2 years with the success criterion of IOP $<21 \mathrm{~mm} \mathrm{Hg}$ with or without medications. Insertion of a GDD was performed without intraoperative complication in all 16 cases. Two patients were eventually diagnosed with uveal melanoma metastases. Metastases were detected 39 months after brachytherapy and 22 months after Ahmed glaucoma valve insertion in 1 patient (case 3) who died 1 month after detection of metastases. The other patient (case 12) was diagnosed with metastases 14 months after brachytherapy and 10 months after Ahmed glaucoma valve insertion. We must emphasize that no patient was found to have local extension to the orbit through the GDD site.

Larger tumor size, ciliary body location, and monosomy 3 are among the strongest risk factors for metastases $[4,34,35]$. At 3 years of follow-up, the cumulative probability of metastasis for tumors with complete chromosome 3 monosomy in a series by Shields et al. [35] was 0 , 24 , and $58 \%$ for small ( $0-3 \mathrm{~mm}$ thickness), medium (3.1$8.0 \mathrm{~mm}$ thickness), and large tumors ( $>8 \mathrm{~mm}$ thickness), respectively; in contrast, the likelihood of metastasis for

Outcomes of GDD Surgery in Eyes with

Treated Uveal Melanoma tumors with disomy 3 was $0,1.4$, and $23.1 \%$ for small, medium, and large tumors during the same time period, respectively. Each millimeter increase in tumor thickness was associated with a $6 \%$ increase in metastasis rate [4]. Forty percent of patients with posterior melanoma will have metastasis in 10 years [36]. Singh and Topham [37] reported that the 5-year survival rate for uveal melanoma was between 76 to $84 \%$. In another report, the mortality rate by any cause in patients with treated uveal melanoma at 5 and 10 year was 19 and 35\%, respectively, and death caused by histopathologically confirmed uveal melanoma at 5 and 10 years was 10 and 17\%, respectively [38]. Our case 3 had ciliary body melanoma with a thickness of $5.86 \mathrm{~mm}$. Case $12 \mathrm{had}$ a tumor thickness of $12.21 \mathrm{~mm}$, monosomy 3 on both FISH and MLPA, and the tumor was located in the choroid. The overall incidence of metastases in our series ( $2 / 16$ or $12.5 \%$ after a median followup of 2.1 years after GDD placement) is similar to that in reports of metastasis in uveal melanoma patients who did not have a GDD.

Case reports exist describing local tumor recurrence and seeding of uveal melanoma in patients who underwent filtration or GDD surgery. Tay et al. [16] performed trabeculectomy on a patient with a history of ciliary body melanoma who had been treated with plaque radiotherapy. The patient had been diagnosed with secondary glaucoma due to multiple mechanisms. Five years after trabeculectomy, he was diagnosed with local treatment failure. The tumor extended to the intraocular lens implant, trabecular meshwork, cornea, and trabeculectomy flap, and there was evidence of extrascleral extension to the conjunctiva. Tan et al. [30] reported the case of a Baerveldt glaucoma implant placed in a patient with iris melanoma who had been treated with proton beam radiation therapy. At 1 year after surgery, the patient had well-controlled IOP with medications and the tumor appeared to be stable. The authors argued that placement of a GDD was promising in terms of controlling IOP, but that patients would need more intensive observation for tumor stability and systemic screening. There are limited case series reporting the outcomes of glaucoma filtration and GDD surgery in patients with malignant melanoma. Sharkawi et al. [17] reported 31 patients with anterior uveal melanoma who were treated with proton beam radiation therapy and who subsequently underwent a Baerveldt glaucoma implant. There were minor complications related to the GDD. The patients demonstrated good IOP control over the 1-year follow-up period with a success rate of $86 \%$. Success was defined as an IOP $\leq 21 \mathrm{~mm} \mathrm{Hg}$ with medications. The investigators did not report any 
metastases or local recurrence in this series. They proposed that GDD surgery could be a good alternative to enucleation in eyes with good visual prognosis or in patients who desire to preserve the eye. The success rate in our study sample was comparable to that of the above study $(80 \%)$ after 1 year. Riechardt et al. [11] reported on the outcomes of trabeculectomy in 15 patients with uveal melanoma treated with proton beam radiation therapy. Two out of 15 patients were diagnosed with local treatment failure. One patient had the eye enucleated and the other patient eventually developed metastases and died 3 years after the diagnosis of iris-ciliary body melanoma. The success rate for IOP control, with the same criteria as ours, was $91 \%$ after 1 year. Four patients (26\%) needed bleb needling and 3 patients (20\%) required complete revision of trabeculectomy [11]. Overall, preliminary evidence to date suggests that glaucoma filtration surgery is an effective approach to control IOP if medical treatment is not adequate; however, local tumor control is a prerequisite for performing any type of filtering or invasive surgery in patients with uveal melanoma.

The metastasis rate of our cohort (13\% within 2 years after placement of a drainage device and a median of 3.5 years after melanoma treatment) is well within the expected range reported in the literature for uveal melanoma patients. The patients who developed metastasis had more aggressive primary tumor biology, which contributed to their demise, rather than having had a glaucoma procedure. Our study is limited, however, by the small size of the sample and the relatively short follow-up time (median of 2 years) after placement of the GDD.

In summary, placement of a GDD is an effective approach for treating elevated IOP and optimizing visual outcomes in eyes treated for ocular melanoma in which there has been no evidence of growth between the time of treatment and tube placement. The findings of our small case series with a median follow-up of 2 years show that placing a GDD in patients with treated uveal melanoma does not expose them to a greater risk of local or extraocular recurrence. However, larger case series with longer follow-up times are required to completely understand and establish the safety of GDDs in patients with treated uveal melanoma.

\section{Acknowledgment}

This work was supported by the George E. and Ruth Moss Trust and by an unrestricted grant from the Research to Prevent Blindness.

\section{Statement of Ethics}

The study was carried out in accordance with the Declaration of Helsinki and the Health Insurance Portability and Accountability Act. An institutional review board waiver for patient consent was obtained for this retrospective review of medical records.

\section{Disclosure Statement}

The authors have no conflicts of interest to declare.

\section{Funding Sources}

This work was supported by the George E. and Ruth Moss Trust and by an unrestricted grant from the Research to Prevent Blindness.

\section{References}

1 Wanner JB, Pasquale LR: Glaucomas secondary to intraocular melanomas. Semin Ophthalmol 2006;21:181-189.

-2 Shields CL, Shields JA, Shields MB, Augsburger JJ: Prevalence and mechanisms of secondary intraocular pressure elevation in eyes with intraocular tumors. Ophthalmology 1987;94:839-846.

- 3 Yanoff M: Glaucoma mechanisms in ocular malignant melanomas. Am J Ophthalmol 1970;70:898-904.

-4 Shields CL, Furuta M, Thangappan A, Nagori S, Mashayekhi A, Lally DR, Kelly CC, Rudich DS, Nagori AV, Wakade OA, Mehta S, Forte L, Long A, Dellacava EF, Kaplan B, Shields JA: Metastasis of uveal melanoma millimeter-by- millimeter in 8,033 consecutive eyes. Arch Ophthalmol 2009;127:989-998.

5 Jensen OA: Malignant melanomas of the uvea in Denmark 1943-1952. A clinical, histopathological, and prognostic study. Acta Ophthalmol (Copenh) 1963;43(suppl 75):1220.

6 Dunnington JH: Intraocular tension in cases of sarcoma of the choroid and ciliary body. Arch Ophthalmol 1938;20:359-363.

7 Marshall CD: On tension in cases of intra-ocular tumour. Trans Ophthalmol Soc UK 1896; 16:155-170.

-8 Gragoudas E, Li W, Goitein M, Lane AM, Munzenrider JE, Egan KM: Evidence-based estimates of outcome in patients irradiated for intraocular melanoma. Arch Ophthalmol 2002;120:1665-1671.

-9 Shields CL, Shields JA, Materin M, Gershenbaum E, Singh AD, Smith A: Iris melanoma: risk factors for metastasis in 169 consecutive patients. Ophthalmology 2001;108:172-178.

10 Girkin CA, Goldberg I, Mansberger SL, Shields JA, Shields CL: Management of iris melanoma with secondary glaucoma. J Glaucoma 2002;11:71-74.

-11 Riechardt AI, Cordini D, Rehak M, Hager A, Seibel I, Boker A, Gundlach E, Heufelder J, Joussen AM: Trabeculectomy in patients with uveal melanoma after proton beam therapy. Graefes Arch Clin Exp Ophthalmol 2016;254: 1379-1385. 
$\checkmark 12$ Damato B, Kacperek A, Chopra M, Sheen MA, Campbell IR, Errington RD: Proton beam radiotherapy of iris melanoma. Int $\mathrm{J} \mathrm{Ra}-$ diat Oncol Biol Phys 2005;63:109-115.

13 Grossniklaus HE, Brown RH, Stulting RD, Blasberg RD: Iris melanoma seeding through a trabeculectomy site. Arch Ophthalmol 1990;108:1287-1290.

-14 Singer PR, Krupin T, Smith ME, Becker B: Recurrent orbital and metastatic melanoma in a patient undergoing previous glaucoma surgery. Am J Ophthalmol 1979;87:766-768.

-15 Sweeney AR, Keene CD, Klesert TR, JianAmadi A, Chen PP: Orbital extension of anterior uveal melanoma after Baerveldt tube shunt implantation. Can J Ophthalmol 2014 49:e133-e135.

16 Tay E, Cree IA, Hungerford J, Franks W: Recurrence of treated ciliary body melanoma following trabeculectomy. Clin Exp Ophthalmol 2009;37:503-505.

17 Sharkawi E, Oleszczuk JD, Bergin C, Zografos L: Baerveldt shunts in the treatment of glaucoma secondary to anterior uveal melanoma and proton beam radiotherapy. Br J Ophthalmol 2012;96:1104-1107.

18 Nath R, Anderson LL, Luxton G, Weaver KA, Williamson JF, Meigooni AS: Dosimetry of interstitial brachytherapy sources: recommendations of the AAPM Radiation Therapy Committee Task Group No. 43. American Association of Physicists in Medicine. Med Phys 1995;22:209-234.

19 Chang MY, Kamrava M, Demanes DJ, Leu M, Agazaryan N, Lamb J, Moral JN, Almanzor R, McCannel TA: Intraoperative ultrasonography-guided positioning of iodine 125 plaque brachytherapy in the treatment of choroidal melanoma. Ophthalmology 2012;119:10731077.
20 McCannel TA, McCannel CA: Iodine 125 brachytherapy with vitrectomy and silicone oil in the treatment of uveal melanoma: 1-to1 matched case-control series. Int J Radiat Oncol Biol Phys 2014;89:347-352.

1 Oliver SC, Leu MY, DeMarco JJ, Chow PE, Lee SP, McCannel TA: Attenuation of iodine 125 radiation with vitreous substitutes in the treatment of uveal melanoma. Arch Ophthalmol 2010;128:888-893.

22 Young TA, Rao NP, Glasgow BJ, Moral JN, Straatsma BR: Fluorescent in situ hybridization for monosomy 3 via 30 -gauge fine-needle aspiration biopsy of choroidal melanoma in vivo. Ophthalmology 2007;114:142-146.

23 Gedde SJ, Herndon LW, Brandt JD, Budenz DL, Feuer WJ, Schiffman JC: Surgical complications in the Tube Versus Trabeculectomy Study during the first year of follow-up. Am J Ophthalmol 2007;143:23-31.e2.

24 Gedde SJ, Schiffman JC, Feuer WJ, Herndon LW, Brandt JD, Budenz DL: Three-year follow-up of the Tube Versus Trabeculectomy Study. Am J Ophthalmol 2009;148:670-684.

25 Minckler DS, Francis BA, Hodapp EA, Jampel HD, Lin SC, Samples JR, Smith SD, Singh K: Aqueous shunts in glaucoma: a report by the American Academy of Ophthalmology. Ophthalmology 2008;115:1089-1098.

26 Shields CL, Materin MA, Shields JA, Gershenbaum E, Singh AD, Smith A: Factors associated with elevated intraocular pressure in eyes with iris melanoma. Br J Ophthalmol 2001;85:666-669.

27 Pasternak S, Erwenne CM, Nicolela MT: Subconjunctival spread of ciliary body melanoma after glaucoma filtering surgery: a clinicopathological case report. Can J Ophthalmol 2005;40:69-71.

28 Durkin SR, Roos D, Higgs B, Casson RJ, Selva D: Ophthalmic and adnexal complications of radiotherapy. Acta Ophthalmol Scand 2007; 85:240-250.
29 Piirtola A, Puska P, Kivela T: Red laser cyclophotocoagulation in the treatment of secondary glaucoma in eyes with uveal melanoma. J Glaucoma 2014;23:50-55.

-30 Tan AN, Hoevenaars JG, Webers CA, Damato B, Beckers HJ: Baerveldt implant for secondary glaucoma due to iris melanoma. Clin Ophthalmol 2010;4:407-409.

31 Ishida K: Update on results and complications of cyclophotocoagulation. Curr Opin Ophthalmol 2013;24:102-110.

32 Egger E, Schalenbourg A, Zografos L, Bercher L, Boehringer T, Chamot L, Goitein G: Maximizing local tumor control and survival after proton beam radiotherapy of uveal melanoma. Int J Radiat Oncol Biol Phys 2001;51:138147.

-33 Egger E, Zografos L, Schalenbourg A, Beati D, Bohringer T, Chamot L, Goitein G: Eye retention after proton beam radiotherapy for uveal melanoma. Int J Radiat Oncol Biol Phys 2003. 55:867-880.

34 Singh AD, Shields CL, Shields JA: Prognostic factors in uveal melanoma. Melanoma Res 2001;11:255-263.

35 Shields CL, Ganguly A, Bianciotto CG, Turaka K, Tavallali A, Shields JA: Prognosis of uveal melanoma in 500 cases using genetic testing of fine-needle aspiration biopsy specimens. Ophthalmology 2011;118:396-401.

36 Shields JA, Shields CL, Donoso LA: Management of posterior uveal melanoma. Surv Ophthalmol 1991;36:161-195.

- 37 Singh AD, Topham A: Survival rates with uveal melanoma in the United States: 19731997. Ophthalmology 2003;110:962-965.

38 Collaborative Ocular Melanoma Study Group: The COMS randomized trial of iodine 125 brachytherapy for choroidal melanoma: V. Twelve-year mortality rates and prognostic factors: COMS report No. 28. Arch Ophthalmol 2006;124:1684-1693. 